\title{
Genetic diversity of hexaploid wheat accessions conserved ex situ at the Japanese gene bank NBRP-Wheat
}

\author{
Nasuda S. ${ }^{1 *}$, Yoshioka M. ${ }^{1}$, Nitta M. ${ }^{1}$, Takenaka S. ${ }^{1,2}$ \\ ${ }^{1}$ Laboratory of Plant Genetics, Kyoto University, Japan \\ ${ }^{2}$ Department of Plant Life Science, Ryukoku University, Japan \\ *e-mail:nasushu@kais.kyoto-u.ac.jp
}

The National BioResource Project (NBRP)-Wheat, the Japanese gene bank of wheat and its relatives, is aimed to promote wheat sciences by providing high quality genetic materials. Currently, the NBRP-Wheat stores more than 12,000 wild species, landraces, and experimental strains of wheat. The main body of collections of wild species and landraces derived from several expeditions Kyoto University have made to the heart of wheat cultivation areas. Some of them are hardly available at the collection sites today. In addition to the primary tasks to maintain the genetic resources, we are surveying genetic diversity among wheat accessions. We established the core collections representing 3,500 hexaplod (AABBDD), 1,900 tetraploid (AABB and AAGG), and 300 diploid (AA) wheat accessions conserved ex situ at NBRP-Wheat. The hexaploid core collection, consisting of 188 accessions of Triticum aestivum, T. spelta, T. compactum, T. sphaerococcum, T. macha, and T. vavilovii, was intensively genotyped by DArTseq markers. Overall, the core collection was divided into seven clusters. Non-admixture accessions in each cluster indicated that the clusters reflect the geographic distribution of the accessions. We analyzed genetic factors controlling seed morphology in hexaploid wheat by utilizing the core collection. The analysis indicated that the hexaploid core collection is a useful genetic tool to dissect complex traits in wheat such as grain morphology analyzed here. Currently we are developing a nested-association mapping (NAM) population representing genetic diversity of East Asian wheat accessions. Twenty four parental accessions, selected form the core collection based on genetic diversity, were crossed with Norin 61 wheat whose genome is de novo sequenced by the International Wheat 10+ Genome Project. The most advanced individual of the NAM population is currently in the $\mathrm{F}_{4}$ generation. Our goal is to prepare and release the East Asian NAM population with more than 3,000 individuals collectively in the $\mathrm{F}_{6}$ or more advanced generations. 